\title{
Bridging the gap between health and non-health investments: moving from cost-effectiveness analysis to a return on investment approach across sectors of economy
}

\section{Pedram Sendi}

Received: 16 June 2006 / Accepted: 29 February 2008 / Published online: 20 March 2008

(C) Springer Science+Business Media, LLC 2008

\begin{abstract}
When choosing from a menu of treatment alternatives, the optimal treatment depends on the objective function and the assumptions of the model. The classical decision rule of cost-effectiveness analysis may be formulated via two different objective functions: (i) maximising health outcomes subject to the budget constraint or (ii) maximising the net benefit of the intervention with the budget being determined ex post. We suggest a more general objective function of (iii) maximising return on investment from available resources with consideration of health and non-health investments. The return on investment approach allows to adjust the analysis for the benefits forgone by alternative non-health investments from a societal or subsocietal perspective. We show that in the presence of positive returns on non-health investments the decision-maker's willingness to pay per unit of effect for a treatment program needs to be higher than its incremental cost-effectiveness ratio to be considered cost-effective.
\end{abstract}

Keywords Cost-effectiveness analysis · Net benefit analysis · Return on investment . Health care costs

JEL Classifications $\quad \mathrm{C} 61 \cdot \mathrm{D} 61 \cdot \mathrm{I} 19 \cdot \mathrm{O} 32$

\section{Introduction}

The idea of using a cost-effectiveness ratio as a cut-off point for resource allocation has been introduced by Weinstein and Zeckhauser (1973) three decades ago. They showed that under conditions of complete divisibility and constant returns to scale independent programs could be ranked according to their average cost-effectiveness ratio and successively implemented, with the most cost-effective program being funded first, until the budget is exhausted

P. Sendi $(\varangle)$

Division of Health Economics, Basel Institute for Clinical Epidemiology, Basel University Hospital, Hebelstrasse 10, 3rd Floor, 4031 Basel, Switzerland

e-mail: pedram.sendi@unibas.ch 
(Weinstein and Zeckhauser 1973). The cost-effectiveness ratio of the last implemented program represents the shadow price of the constrained budget or the threshold ratio above which programs are considered to be cost-ineffective (Weinstein and Zeckhauser 1973; Weinstein 1995). However, in the presence of mutually exclusive programs the incremental cost-effectiveness ratio rather than the average cost-effectiveness ratio should be used to determine whether a program is cost-effective or not (Weinstein 1995; Karlsson and Johannesson 1996). Programs that are dominated, i.e. that cost more and are less effective than the comparison program for the same disease condition, are never implemented. This decision rule follows from a linear programming approach to solving a constrained optimisation problem where the objective is to maximise health outcomes subject to a budget constraint (Stinnett and Paltiel 1996; Birch and Gafni 1992, 1993).

An alternative approach to determining whether a program should be implemented or not is the net monetary benefit (NMB) approach, which was introduced because of its attractive statistical properties to improve the way uncertainty is handled in cost-effectiveness analysis (Stinnett and Mullahy 1998; Tambour et al. 1998; Briggs and Gray 1999). The NMB represents a linear transformation of incremental costs and effects, where the effectiveness units are multiplied by the price per effectiveness unit and the costs subtracted thereof (Tambour et al. 1998). A program with a positive incremental monetary benefit is worth implementing. An attractive property of the NMB is that the difference between the average NMBs between two programs is equivalent to the incremental NMB (Stinnett and Mullahy 1998; Tambour et al. 1998). This also facilitates the comparison of multiple mutually exclusive programs as the program with the highest average NMB is the program that should be chosen (Briggs et al. 2002).

The decision rules of cost-effectiveness analysis have hitherto focused on the allocation of scarce resources within the health care sector. Agencies at the societal or subsocietal level, however, may also want to consider return on non-health investments in decision making. A societal decision-maker, for example the government in a publicly funded health care system, may have to decide what proportion of the budget should be invested in health care programs and what proportion in other areas such as transportation or education. The same holds true for subsocietal decision-makers, who have to meet budgetary constraints. The most extreme case of a subsocietal decision-maker is the patient. In Switzerland, for example, patients usually pay the whole amount for dental care with out-of-pocket money. Consequently, consideration must be given to the opportunity cost of resources invested in dental care, which are usually in areas other than health.

In this paper we suggest an investment approach to budget allocation that represents a modified net benefit framework and builds a bridge between health and non-health investments to maximise return on investment from available resources. In the next section, we review the classical decision rule of cost-effectiveness analysis. In the section "The net benefit approach", we illustrate that the net benefit approach implies a different objective function and different constraints. In the penultimate section we suggest a modified net benefit approach for choosing between mutually exclusive programs when the opportunity cost of resources may be in areas other than health. Finally, we conclude by a discussion of our approach.

\section{The classical decision rule of cost-effectiveness analysis}

Consider the four mutually exclusive programs shown in Table 1 . These programs may represent four different prosthetic treatment alternatives for edentulous patients. Program A1 could, for example, represent no intervention, i.e. the costs and effects associated with the continuous use of the current complete denture, which might be suboptimal. Program A2 
Table 1 Cost per patient $(C)$, effect per patient $(E)$, and incremental cost-effectiveness ratio per patient $(\Delta C / \Delta E)$ for four hypothetical treatment alternatives

\begin{tabular}{lllll}
\hline Treatment & $C(\$)$ & $E$ & $\Delta C / \Delta E$ & $\Delta C / \Delta E^{\mathrm{a}}$ \\
\hline A1 & 200 & 20 & 10 & 10 \\
A2 & 300 & 25 & 20 & 17 \\
A3 & 400 & 32 & 14 & 33 \\
A4 & 500 & 35 & 33 & \\
\hline
\end{tabular}

a After exclusion of treatment alternatives by extended dominance (Weinstein 1995)

could represent a new complete denture, program A3 an implant-retained overdenture prosthesis using two implants in the mandible (i.e. lower jaw), and program A4 could represent an implant-supported overdenture-prosthesis using four implants in the mandible. The cost and effect estimates in Table 1 are purely hypothetical and not critical to make our conceptual point. However, a useful outcome measure in prosthodontics could be, for example, quality-adjusted prosthesis-years (QAPYs) (Sendi et al. 1997; Zitzamnn et al. 2006).

Lets assume that dental care would be part of the publicly funded health care package as is currently the case in Germany and the objective would be to maximise the dental health of the edentulous population, given the available budget. This represents a constrained optimisation problem that may be approached using linear programming. The optimisation problem can be formulated as follows:

$$
\begin{array}{ll}
\text { maximise: } & \sum x_{i} e_{i} \\
\text { subject to: } & 0 \leqslant x_{i} \leqslant 1000 \quad(\text { for all } i) \\
& \sum x_{i} c_{i} \leqslant C \\
& \sum x_{i} \leqslant 1000 \quad(\text { for } i \in \mathrm{A})
\end{array}
$$

where $e_{i}$ and $c_{i}$ denote the effect and cost of treatment $i$ per patient, $C$ denotes the budget, $x_{i}$ denotes the number of patients to whom treatment $i$ is provided, and $A$ denotes a set of mutually exclusive treatments.

The solution to this optimisation problem is shown in Table 2 for the budget levels of $\$ 100,000$ to $\$ 500,000$. Program A2 is excluded by extended dominance and is therefore not provided to any patient. For example, at the budget level $\$ 300,000$, total aggregate effects are highest when 500 patients receive A1 (i.e. no replacement of the suboptimal current complete denture) and 500 patients receive A3 (i.e. implant-retained overdenture prosthesis).

This may raise ethical issues (Sendi 2002; Sendi and Al 2003). In a publicly funded health care system it might be seen as unacceptable that some patients receive preferential treatment while other patients receive no treatment at all, i.e. programs may be considered indivisible for ethical reasons (Sendi 2005). The budget as a decision rule in a linear programming framework might therefore violate the ethical preferences of the general public. Integer programming for constrained optimisation handles health care programs as indivisible units and therefore provides a solution to this disadvantage of the linear programming approach. If the optimisation problem above would be approached using integer programming (i.e. if programs would be handled as completely indivisible), the program that would be funded at the budget level $\$ 300,000$ would be A2 since this is the program with the highest aggregate health outcome that can be implemented completely (Birch and Gafni 1992, 1993). 
Table 2 Number of patients to be treated in each treatment group in order to maximise aggregate effects subject to the budget constraint

\begin{tabular}{|c|c|c|c|c|c|}
\hline \multirow[t]{2}{*}{ Budget $^{\mathrm{b}}$} & \multicolumn{5}{|c|}{ Number of patients per treatment ${ }^{a}$} \\
\hline & A1 & A2 & A3 & A4 & Aggregate effects ${ }^{\mathrm{c}}$ \\
\hline 100 & 500 & & & & 10,000 \\
\hline 200 & 1,000 & & & & 20,000 \\
\hline 300 & 500 & & 500 & & 26,000 \\
\hline 400 & & & 1,000 & & 32,000 \\
\hline 500 & & & & 1,000 & 35,000 \\
\hline
\end{tabular}

a There are 1,000 patients eligible for treatment

b Budget in $\$ 1,000$

c $\sum x_{i} e_{i}$

\section{The net benefit approach}

An alternative to the budget as a decision rule is the decision maker's maximum willingness to pay per unit of effect (e.g. QALY or QAPY). Lets assume that the government is the decision maker, this is the most appropriate perspective for an economic evaluation in dental care in Germany since the costs are usually covered by public health insurance. In this case the budget for dental care would be determined ex post at the end of the year and is hence not an optimisation constraint as described in the section above (Karlsson and Johannesson 1996). The optimisation problem can therefore be written as:

$$
\begin{aligned}
\text { maximise: } & \sum\left(e_{i} \lambda-c_{i}\right) x_{i} \\
\text { subject to: } & x_{i}=1 \text { or } 0 \quad(\text { for all } i) \\
& \sum x_{i}=1 \quad(\text { for } i \in \mathrm{A})
\end{aligned}
$$

where $e_{i}$ and $c_{i}$ denote the effect and cost of treatment program $i, x_{i}$ is the decision variable and indicates whether treatment program $i$ is chosen, $A$ denotes a set of mutually exclusive treatment programs, and $\lambda$ the decision maker's willingness to pay per additional unit of effect. The value of $\lambda$ may be conditioned on other variables such as severity of disease or magnitude of health benefit. This integer programming approach maximises the NMB or net present value (Al et al. 2004), which is defined as $e_{i} \lambda-c_{i}$ under (5). Note that $\lambda$ in this case is not the ex ante shadow price of the constrained budget since the budget is determined ex post. Rather, it is a subjective measure of the value of dental care to the decision-maker and a fixed value (Al et al. 2004). However, it is the shadow price of the decision-maker's ex post budget used for dental care.

The NMB of the four treatment alternatives as a function of $\lambda$ are shown in Fig. 1. The preferred treatment is the one that offers the highest average NMB (Briggs et al. 2002). The cut-off points where one treatment becomes preferable to another treatment coincides with the incremental cost-effectiveness ratio of the respective treatment (Briggs et al. 2002). Note that when the net benefit approach is taken, treatments that are excluded by extended dominance (Karlsson and Johannesson 1996) will never represent the optimal solution to the constrained optimisation problem. This is because the budget is determined after the decision maker's willingness to pay per unit of effect has been determined and indivisibility of programs is therefore not a problem.

The approach of maximising the net present value using a fixed threshold for the costeffectiveness ratio is actually the most widely used method for decision making in health care 


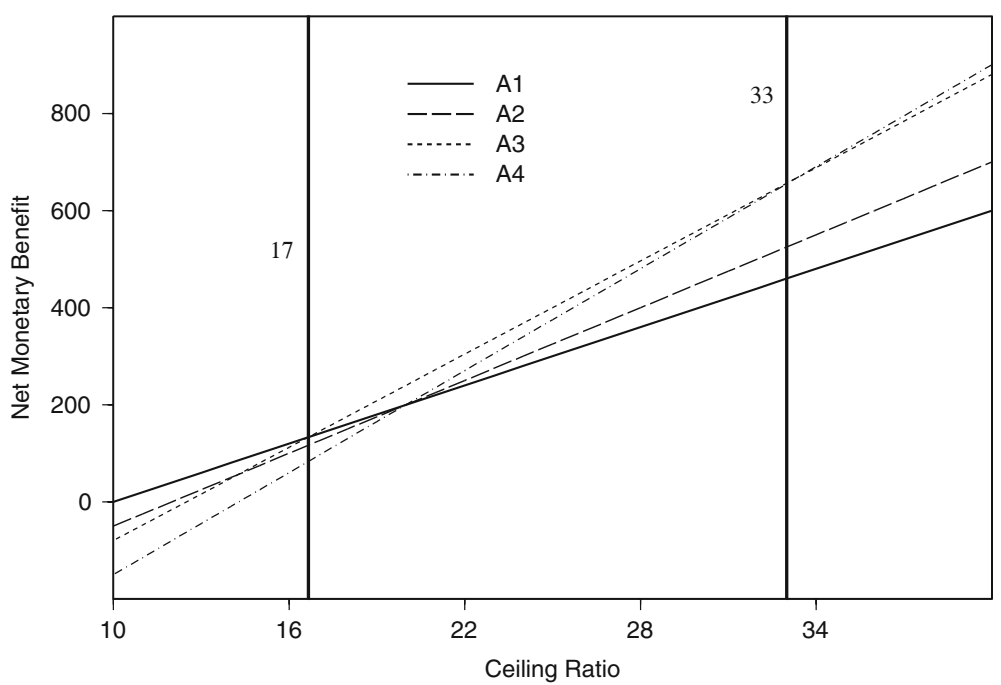

Fig. 1 NMB of four mutually exclusive interventions as a function of the decision maker's willingness to pay per unit of effect (ceiling ratio)

as a real world budget does not exist from a societal perspective (Sendi 2005). The absence of a societal real world budget therefore technically precludes the budget as decision rule as described above (Sendi 2005). However, an analysis of reimbursement recommendations of NICE in the UK, for example, shows that most decisions are not based on a single fixed threshold value for determining cost-effectiveness but on a range of threshold values (Devlin and Parkin 2004). This is in line with a recent survey of decision-makers' goals in health care that stressed the importance of maximising the net present value using severity weighted health effects from the societal perspective (Al et al. 2004).

\section{An investment perspective}

The constrained optimisation problems described above either maximise health outcomes subject to the budget constraint or maximise the net benefit of the intervention given a decision maker's willingness to pay per unit of effect with the budget being determined ex post. A limitation of the first approach is that the most expensive and effective dental treatment will be selected if the budget will be large enough to fund that treatment. However, decision makers (be it the Ministry of Health or the patient) may not necessarily want to finance the most expensive treatment options even though the available budget would allow them to do so. The net benefit approach, on the other hand, respects the decision maker's subjective valuation of the benefit of a certain treatment but does not explicitly consider the opportunity costs of the additional resources needed to invest in a more expensive treatment alternative, which may be in areas other than health (e.g. financial investments).

An alternative approach to choosing one of the four mutually exclusive treatment alternatives is to take an investment perspective. The idea is to account for the fact that the opportunity cost of resources for dental care may be in areas other than health. If the budget is spent on more expensive dental care, other investment opportunities are forgone. In a publicly funded dental health care system the opportunity cost of health care resources could, 
e.g. be in areas such as education or transportation. It seems natural to include these forgone opportunities in decision making. This requires, however, that return on investment in dental health is measured in the same units as in non-health investments.

Return on investment in health can be expressed as NMB/C, i.e. the $\mathrm{NMB}$ of a treatment divided by its costs, as described in more detail elsewhere (Sendi et al. 2004a,b). The results can then be expressed in percentages and this allows us to build a bridge between investments in health care and non-health investments (Sendi etal. 2004a,b). If the objective is to maximise overall return on investment, the constrained optimisation problem can be written as:

$$
\begin{aligned}
\text { maximise: } & \sum w_{1 i} x_{i}\left(\frac{e_{i} \lambda-c_{i}}{c_{i}}\right)+w_{2 i} x_{i} r \\
\text { subject to: } & x_{i}=1 \text { or } 0 \quad(\text { for all } i) \\
& \sum x_{i}=1 \quad(\text { for } i \in A) \\
& \sum x_{i} c_{i} \leq B
\end{aligned}
$$

where $e_{i}$ and $c_{i}$ denote the effect and cost of treatment program $i, x_{i}$ is the decision variable and indicates whether treatment program $i$ is chosen, $A$ denotes a set of mutually exclusive treatment programs $\lambda$ denotes the decision maker's willingness to pay per marginal unit of effect, $B$ the total budget ( $\$ 1,000$ per patient in our hypothetical example), $r$ the return if the budget would be invested in areas other than health (10\% in our hypothetical example) and $w_{1 i}=\frac{c_{i}}{B}$ (the proportion of the budget invested in treatment $i$ ) and $w_{2 i}=\frac{B-c_{i}}{B}$ (i.e. the proportion of the budget invested in areas other than health if treatment $i$ is chosen). The optimal solution will be the intervention that maximises return on investment from available resources, given the decision maker's maximum willingness (WTP) to pay per unit of effect. Figure 2 shows the returns on investment in the four treatment options listed in Table 1 for a budget of $\$ 1,000$ per patient. At a low WTP per unit of effect treatment A1 is the preferred treatment option. With a WTP of more than 18.5 per unit of effect, treatment A3 becomes the preferred strategy. And with a WTP of more than 37 per unit of effect, treatment A4 becomes the preferred treatment option.

Importantly, note that the objective function (8) simplifies to maximising $\sum x_{i} \frac{e_{i} \lambda-c_{i}}{B}$ if we assume that the budget is available for dental health care only, without consideration of non-health investments (i.e., $w_{2 i}=0$ ). Equation 8 is then simply a linear transformation of the NMB (i.e. the NMB divided by the budget $\mathrm{B}$ ) and the ceiling ratios where one program becomes preferable to the other would exactly coincide with the ceiling ratios (i.e. incremental cost-effectiveness ratios) if the NMB is maximised. That is, if we assume $w_{2 i}=0$, then the solutions to maximising Eqs. (5) or (8) are exactly the same for a given WTP per marginal unit of effect. Note that the difference between the investment approach and the net benefit approach lies in an objective function (8) extended for non-health investments and the additional budget constraint (11).

However, the higher the return on investments in areas other than dental health (i.e. the higher $r$ ), the higher the WTP per unit of effect needs to be to render A2 or A4 the preferred strategy. This is because the opportunity cost of the additional resources needed for more expensive (and more effective) treatments are the forgone returns that could have been achieved with non-dental health investments, with returns higher than zero. Investments in health care now have to compete with the return on investment in areas other than health. This is also clear from the figures where the cut-off levels are slightly higher in Fig. 2 than in Fig. 1. In our hypothetical example, we assumed a return of $10 \%$ for investments other than 


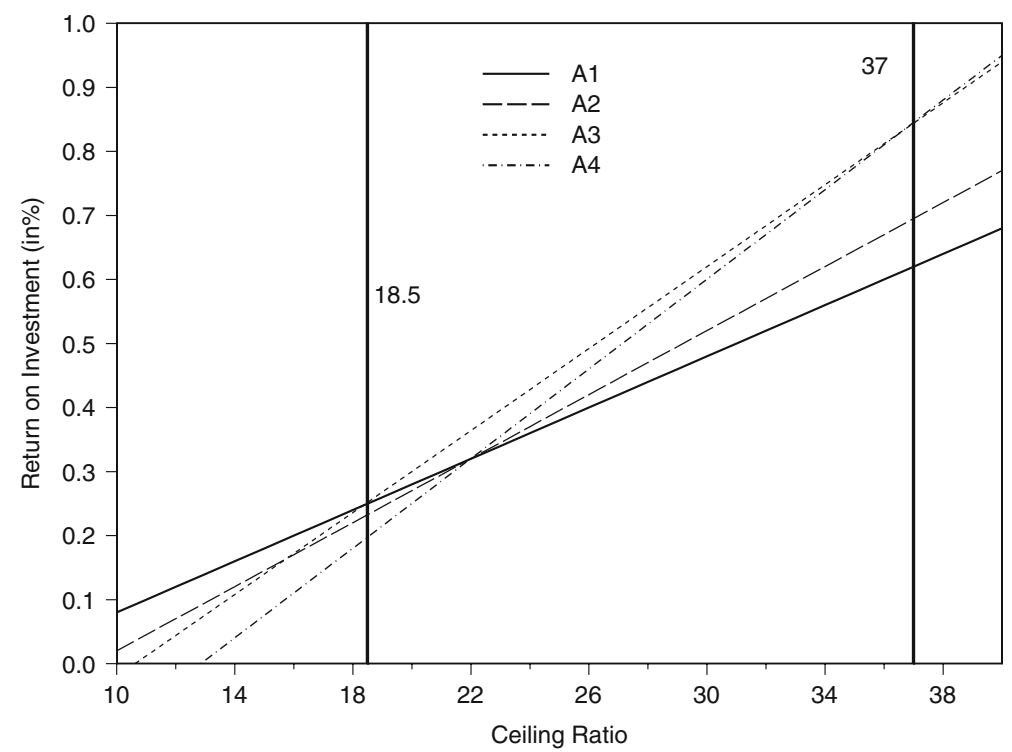

Fig. 2 Return on investment in four mutually exclusive interventions as a function of the decision maker's willingness to pay per unit of effect (ceiling ratio) (Return on investment has been calculated for the four programs listed in Table 1 with a budget of $\$ 1,000$ per patient, and a return on investment of $10 \%$ if the money is invested in non-dental care)

dental care. With a return of $50 \%$ in non-dental care investments and a budget of $\$ 1,000$ per patient, the cut-off levels where treatment A2 and A4 would become the optimal choices are 30 and 50, respectively.

\section{Discussion}

In a cost-effectiveness analysis the results are typically expressed as incremental costeffectiveness ratios that inform about the extra resources needed for an additional unit of effect (Karlsson and Johannesson 1996). The net benefit approach, on the other hand, has been suggested as an alternative way of presenting the results of a cost-effectiveness analysis (Stinnett and Mullahy 1998; Tambour etal. 1998). Instead of calculating the incremental cost-effectiveness ratio between mutually exclusive treatments, the average NMB can be calculated and the intervention with the highest average net benefit represents the optimal solution (Briggs et al. 2002).

We have shown that if the objective is to maximise health outcomes subject to a budget constraint (in an integer programming framework assuming complete indivisibility), then treatments that would have been excluded by extended dominance in a linear programming framework may happen to represent the optimal solution. However, if the objective of the decision-maker is to maximise the net benefit of an intervention given his WTP per unit of effect, then the budget must be determined ex post so that the complete program can be financed. In this case programs that are excluded by extended dominance are never implemented. The cost-effectiveness ratio (or the NMB) as a decision rule therefore requires a flexible budget. 
However, decision-makers may have a budget larger than the costs of the most expensive and effective dental treatment but may be reluctant to actually finance the most expensive program. That is, they implicitly weight the costs versus the benefits of the treatment in a subjective manner, which is actually expressed as the patient's willingness to pay per unit of effect. In addition, for policy making across sectors of economy it is necessary to incorporate in the model the opportunity cost of the additional budget required for more expensive treatments, which are the returns forgone in investments other than health care. We therefore suggest to express the return on investment in health care in terms of percentages that would allow us to maximise the joint return from health and non-health investments.

The implication of our approach is that with a positive rate of return in investments other than health care, the decision-maker's WTP per unit of effect needs to be higher than the incremental cost-effectiveness ratio of the respective treatment alternative to be considered cost-effective. The higher the return on non-health investments will be, the higher the decision maker's WTP per unit of health effect needs to be compared to the incremental cost-effectiveness ratio to warrant adoption. This has important implications for decision-making and may suggest that incremental cost-effectiveness ratios may overestimate the cost-effectiveness of interventions when non-health investments are considered from a broader perspective. Note that the net benefit approach is equivalent to the investment approach when non-health investments are ignored. However, explicitly considering non-health investments in addition to health investments leads to a different objective function as demonstrated above.

In this paper we have presented the extended net benefit framework under conditions of certainty. However, costs and effects of health care interventions are inherently uncertain. The overall return on investment as defined under (8) is therefore also subject to uncertainty. The presence of uncertainty associated with costs and effects therefore introduces additional statistical issues in decision making that need to be addressed by future research.

In conclusion, we believe that the investment approach represents a useful addition to the current armamentarium in economic evaluation and may help to improve the efficiency in the allocation of resources across sectors of economy. This may be of particular relevance in countries with a social health care system.

Acknowledgements I am grateful to Bruce Craig for his comments on an earlier draft of this article.

\section{References}

Al, M. J., Feenstra, T., \& Brouwer, W. B. (2004). Decision makers' views on health care objectives and budget constraints: Results from a pilot study. Health Policy, 70, 33-48.

Birch, S., \& Gafni, A. (1992). Cost effectiveness/utility analyses. Do current decision rules lead us to where we want to be? Journal of Health Economics, 11, 279-296.

Birch, S., \& Gafni, A. (1993). Changing the problem to fit the solution: Johannesson and Weinstein's (mis) application of economics to real world problems. Journal of Health Economics, 12, 469-476.

Briggs, A. H., Goeree, R., Blackhouse, G., \& O’Brien, B. J. (2002). Probabilistic analysis of cost-effectiveness models: Choosing between treatment strategies for gastroesophageal reflux disease. Medical Decision Making, 22, 290-308.

Briggs, A. H., \& Gray, A. M. (1999). Handling uncertainty when performing economic evaluation of healthcare interventions. Health Technology Assessment, 3, 1-134.

Devlin, N., \& Parkin, D. (2004). Does NICE have a cost-effectiveness threshold and what other factors influence its decisions? A binary choice analysis. Health Economics, 13, 437-452.

Karlsson, G., \& Johannesson, M. (1996). The decision rules of cost-effectiveness analysis. Pharmacoeconomics, 9, 113-120. 
Sendi, P. (2002). Some reflections on cost-effectiveness analysis and budget allocation in medicine. Expert Review of Pharmacoeconomics and Outcomes Research, 2, 191-193.

Sendi, P. (2005). Decision rules and uncertainty in the economic evaluation of health care technologies. PhD Disseration, Erasmus University Rottredam.

Sendi, P., \& Al, M. J. (2003). Revisiting the decision rule of cost-effectiveness analysis under certainty and uncertainty. Social Science and Medicine, 57, 969-974.

Sendi, P., Al, M. J., \& Rutten, F. F. H. (2004a). Portfolio theory and cost-effectiveness analysis: A further discussion. Value Health, 7, 595-601.

Sendi, P., Al, M. J., \& Zimmermann, H. (2004b). A risk-adjusted approach to comparing the return on investment in health care programs. International Journal of Health Care Finance and Economics, 4, 199-210.

Sendi, P. P., Palmer, A. J., \& Marinello, C. P. (1997). Health state utilities in dentistry. A review. Acta Med Dent Helvetica, 2, 243-248.

Stinnett, A. A., \& Mullahy, J. (1998). Net health benefits: A new framework for the analysis of uncertainty in cost-effectiveness analysis. Medical Decision Making, 18, S68-S80.

Stinnett, A. A., \& Paltiel, A. D. (1996). Mathematical programming for the efficient allocation of health care resources. Journal of Health Economics, 15, 641-653.

Tambour, M., Zethraeus, N., \& Johannesson, M. (1998). A note on confidence intervals in cost-effectiveness analysis. International Journal of Technology Assessment in Health Care, 14, 467-471.

Weinstein, M. C. (1995). From cost-effectiveness ratios to resource allocation: Where to draw the line? In F. A. Sloan (Ed.), Valuing health care. Cambridge UK: Cambridge University Press.

Weinstein, M. C., \& Zeckhauser, R. J. (1973). Critical ratios and efficient allocation. Journal of Public Economics, 2, 147-157.

Zitzamnn, N. U., Marinello, C. P., \& Sendi, P. (2006). A cost-effectiveness analysis of implant overdentures. Journal of Dental Research, 85, 717-721. 